\title{
Decomposição de Ondaletas, Análise de Volatilidade e Correlação para İndices Financeiros ${ }^{\star}$
}

\author{
Edgard Almeida Pimentel ${ }^{\star}$ \\ Juliana Fernandes da Silva**
}

\begin{abstract}
Resumo
Acontecimentos recentes no ambiente financeiro internacional suscitaram algumas questões relacionadas à inter-relação e interdependência dos diversos mercados ao longo do globo e seus graus de integração. Nesse sentido, este artigo propõe uma análise de variância e correlação para índices financeiros como o Dow Jones Industrial, o Ibovespa e o Euro Stoxx 50 através da decomposição destas séries em ondaletas. Uma vez que a metodologia de ondaletas é capaz de separar as diferentes frequências de uma série temporal ao longo do tempo (frequência-temporal), o estudo de uma estrutura de correlações e variâncias através desta metodologia é capaz de evidenciar fenômenos particulares de cada frequência de dados que, de forma agregada, são perdidos.
\end{abstract}

\section{Palavras-Chave}

ondaletas, séries financeiras, análise de volatilidade e correlação

\section{Wavelets Decomposition, Volatility Analysis and Correlation for Financial Indexes}

\begin{abstract}
Since the benefits of portfolio international diversification results appeared in financial literature, the issue of financial co-movements among different markets has been a key question. Many recent studies have approached this topic targeting a correlation investigation among financial indicators. Unfortunately, the huge majority of them focus only in the time domain, ignoring the relevant frequency domain which could differentiate long and short-run investment contribution to the energy of a time series. This paper proposes a wavelet-based volatility and correlation analysis of key financial indexes for the Brazilian, American and European markets looking for some results concerning the correlation structure between them in time and frequency domain, obtaining distinct results for long and short-run investment performance and contribution.
\end{abstract}

\section{Keywords}

wavelets, financial time-series, correlation and volatility analysis, comovements

\section{JEL Classification}

C10, G10, G12

\footnotetext{
- Artigo recebido em outubro de 2009 e aceito para publicação em outubro de 2010. Os autores agradecem ao Prof. Pedro Alberto Morettin por comentários enriquecedores a uma versão anterior deste trabalho. São também gratos às sugestões de um parecerista anônimo.

* Instituto Superior Técnico (UTL)- Universidade Técnica de Lisboa - Doutorando em Matemática do Departamento de Matemática. Fellow da European Comission for Education and Training (Grant \#L15 a0900194) - Endereço para contato: Av. Rovisco Pais 1 - 1049-001 - Lisboa - Portugal Email: epiment@math.ist.utl.pt

** Instituto Superior Técnico (UTL)- Universidade Técnica de Lisboa - Doutoranda em Matemática do Departamento de Matemática. Fellow da European Comission for Education and Training (Grant \#L15 a0900 196) - Endereço para contato: Av. Rovisco Pais 1 - 1049-001 - Lisboa - Portugal Email: jusilva@math.ist.utl.pt
} 


\section{Introdução}

O estudo da co-movimentação entre índices financeiros, ou de sua correlação, é de largo interesse no estudo de finanças internacionais. Por um lado, apresenta informações altamente relevantes do ponto de vista do gerenciamento de risco, bem como da perspectiva da diversificação de portfólios. ${ }^{1}$

Pela importância do tema, uma série de estudos tem abordado a noção de correlação entre ativos financeiros de diversos mercados, buscando entender como o movimento dos retornos destes ativos relacionam-se (ver, por exemplo, [Ki94], [Li94], [LoSo95], [LoSo01], [KaSt96], [FoRi02], [BrDe05], [BrDe06]). Dentre esses estudos, as metodologias utilizadas são, na sua totalidade, destinadas à análise da correlação entre os retornos abordados na dimensão temporal, ou seja, como a correlação entre os retornos comporta-se ao longo do período de tempo sob análise.

Entretanto, além da dimensão temporal, uma informação financeira agregada carrega componentes de diferentes frequências, i.e., investimentos de frequência mais alta e mais baixa, longo e curto prazos. Claramente, o grau de correlação de mercados distintos deve variar entre frequências distintas. Um estudo bastante recente nesse sentido é [RuNu09].

O mesmo deve ocorrer para medidas de volatilidade. Analisar a volatilidade de uma particular série de retornos sem considerar diferentes frequências pode colapsar as contribuições de investimentos de curto e longo prazos numa única componente, o que gera imprecisões nos resultados de análise.

Assim sendo, este trabalho propõe a análise de correlação entre índices financeiros relativos aos mercados americano, europeu e brasileiro, precisamente o Dow Jones Industrial, Euro Stoxx 50 e Ibovespa para os últimos cinco anos, estudando a estrutura de correlação entre estas séries, bem como procedendo a uma análise de volatilidade por meio da metodologia de ondaletas. Além disso, o estudo ainda investiga o comportamento de cada série por meio de uma transformada discreta de ondaletas e da análise de multiresolução, empregada através da transformada discreta de ondaletas de máxima sobreposição. Assim, como resultados, além das estruturas de variância e correlação, o artigo ainda discorre sobre as principais componentes e suas contribuições em fenômenos como a recente turbulência financeira.

A segunda seção do artigo discute o banco de dados utilizado, bem como introduz os conceitos de ondaletas e a metodologia utilizada. A terceira seção apresenta algu-

l Para uma análise dos benefícios da diversificação internacional de portfólios, referimo-nos a [Gr68]. 
mas estatísticas acerca dos índices estudados, assim como implementa uma análise exploratória baseada na metodologia de ondaletas. Uma quarta seção apresenta os resultados para a análise de correlações e variâncias, enquanto uma seção final discute algumas conclusões.

\section{Metodologia e Bases de Dados}

\subsection{Bases de Dados}

Os dados utilizados neste trabalho são os retornos ${ }^{2}$ financeiros das séries financeiras Ibovespa, Euro Stoxx 50 e Dow Jones Industrial, no período de 10/11/2004 até 5/06/2009. Por se tratar de informações diárias, os dados foram compatibilizados de modo que houvesse 1024 observações para cada série e, mais ainda, que todas essas observações fossem referentes à mesma data para cada série. Assim, a compatibilização levou em consideração as diferenças entre os dias de funcionamento dos mercados financeiros na Europa, Estados Unidos e Brasil.

A escolha por estes índices foi baseada na capacidade destes de avaliarem, ou representarem, o desempenho de cada um dos mercados sob análise, o que parece razoável dada a relevância de cada um deles. O Euro Stoxx 50 congrega cinquenta ativos de empresas consideradas líderes em setores-chave para a economia da zona do Euro, espalhadas em doze países da União Européia. Já o Ibovespa e o Dow Jones são índices classicamente tomados em consideração quando da análise financeira nos ambientes brasileiro e americano.

O uso de 1024 observações deve-se à metodologia de ondaletas empregada, e será discutida na subseção seguinte.

\subsection{O Método de Ondaletas}

Ondaletas são funções. Seu uso na decomposição de um sinal, ou série temporal, tem uma motivação no fato de que este sinal pode ser entendido como um elemento de um espaço de Hilbert, a saber, o $L_{2}$, o espaço das funções de quadrado somável sobre R. Como é um fato largamente conhecido, todo espaço de Hilbert possui uma base ortonormal, em termos da qual seus elementos podem ser escritos. No caso do $L_{2}$, é possível obtermos uma base de ondaletas.

2 O retorno do ativo $i$ no instante $t$ é dado por: $r_{i, t}=\ln \left(x_{i, t}\right)-\ln \left(x_{i, t-1}\right)$, onde $x_{i, t}$ é o preço do ativo $i$ no instante $t$. 
A noção de ondaletas foi introduzida em 1910 por Haar [Hal0] e recentemente desenvolvida em trabalhos como [Gr88] e [Da92]. Algumas classes de ondaletas podem ser mencionadas, como a ondaleta Meyer, Morlet e o chapéu mexicano. Na Figura 1, abaixo, vemos o gráfico de uma ondaleta:

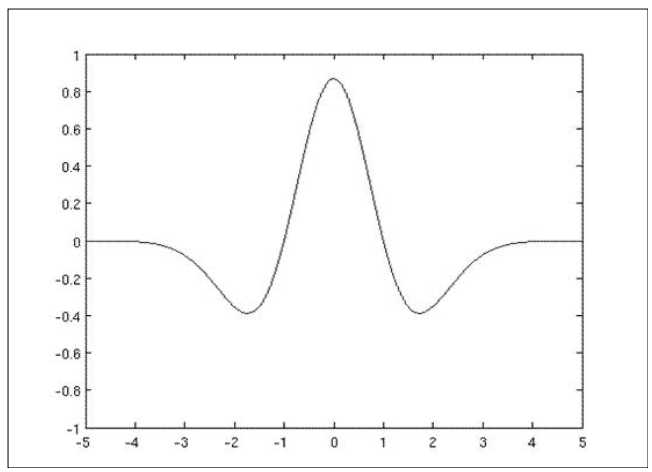

Figura 1 - Exemplo de Ondaleta, o Chapéu Mexicano

Através do estudo dos coeficientes de um dado sinal em termos de uma base de ondaletas, obtemos informações acerca da série temporal. Em termos bastante gerais, a ideia por trás da análise de ondaletas é entender como uma particular média ponderada de um conjunto de funções varia entre períodos.

Em se tratando de uma série temporal, este intuito pode ser traduzido como o entendimento de como os componentes do sinal variam em diferentes escalas, ou seja, em diferentes frequências temporais. Assim, por exemplo, ao analisarmos a série de retornos do Ibovespa através da metodologia de ondaletas, podemos endereçar questões como a diferença de volatilidade entre os investimentos especulativos e o investimento em bolsa de micro e pequenos investidores.

Formalmente, temos a:

Definição 1 - Seja $\varphi: \mathrm{R} \rightarrow \mathrm{R}$ tal que

$$
\int_{-\infty}^{\infty} \varphi(u) d u=0 e \int_{-\infty}^{\infty} \varphi^{2}(u) d u=1
$$

Então, $\varphi: R \rightarrow R$ é chamada ondaleta. Se, ainda, a trasformada de Fourier de $\varphi$,

$$
\Phi(f)=\int_{-\infty}^{\infty} \varphi(u) \exp (-i 2 \pi f(u)) d u \mathrm{t}
$$


for tal que $C_{\Phi} \equiv \int_{-\infty}^{\infty} \frac{\left|\Phi^{2}(f)\right|}{f} d f$

satisfaz $0<C_{\Phi}<\infty$, então $\varphi$ é dita uma ondaleta admissível.

Em uma dada família de ondaletas, por exemplo, a família Haar ou Morlet, dois tipos de funções podem ser mencionadas, as ondaletas pai e ondaletas mãe. As primeiras são, de modo geral, empregadas nos componentes de mais alta frequência, ao passo que as ondaletas mãe são utilizadas nos componentes de frequência mais baixa. De forma alternativa, pode-se dizer que as ondaletas pai endereçam componentes de tendência da série temporal, enquanto as ondaletas mãe encarregam-se dos componentes responsáveis por desvios com relação à tendência.

Assim, dada uma ondaleta pai $\phi$, uma ondaleta mãe $\varphi$, e um inteiro positivo $J$, pode-se obter uma família:

$$
\left\{\phi_{j, k}, \varphi_{j, k}, j=1,2, \ldots ; k=1,2, \ldots\right\}
$$

cujos elementos são:

$$
\phi_{j, k}(t)=2^{-\frac{1}{2} \phi}\left(\frac{t-2^{j} k}{2^{j}}\right)
$$

e

$$
\varphi_{j, k}(t)=2^{-\frac{1}{2}} \varphi\left(\frac{t-2^{j} k}{2^{j}}\right)
$$

Desta forma, dado um elemento $f \in L_{2}(R)$, construímos as projeções ortogonais:

$$
\bigcup_{J, k}=\int f(t) \phi_{j, k}(t) d t
$$

e

$$
\omega_{j, k}=\int f(t) \varphi_{j, k}(t) d t
$$


Então, $f(t)$ pode ser escrita como:

$$
f(t)=\sum_{k} \bigcup_{J, k} \phi_{J, k}(t)+\sum_{j=1}^{J-1} \sum_{k} \omega_{j, k} \varphi_{j, k}(t)
$$

A expressão em (1) é a representação da função $f$ em uma base de ondaletas. É clara a semelhança entre a representação em (1) e a representação de uma função de $L_{2}(0,2 \pi)$, dada em termos dos coeficientes de Fourier e de funções trigonométricas.

De posse da definição formal de uma ondaleta, passemos à discussão da transformada discreta de ondaletas (TDO) e da transformada discreta de ondaletas de máxima sobreposição (TDOMS). Vejamos primeiramente o caso da TDO.

Considere uma série temporal de tamanho $N=2^{J}$ dado por $X$. Então, o vetor da transformada discreta de ondaletas, $\omega$, de comprimento $N$ é dado por:

$$
\omega=W X
$$

onde $W$ é a matriz do $\mathrm{R}^{\mathrm{N}_{x} \mathrm{~N}}$ que define a TDO de $X$. A matriz pode ser escrita na forma:

$$
\omega=\left[\omega_{1}, \ldots, \omega_{J}, U_{J}\right]
$$

onde cada $\omega_{i}$ é um vetor de tamanho $N / 2^{i}$ associado a mudanças na escala $\sigma_{i}=2^{i-1}$, enquanto $\bigcup_{J}$ é um vetor de comprimento $\mathrm{N} / 2^{\mathrm{J}}$ e está associado aos coeficientes referentes às médias da escala $2^{J}$. Já a matriz ortonormal $W$ consiste dos filtros - ou ondaletas - utilizados na decomposição do sinal.

Passemos agora à análise da transformada discreta de ondaletas de máxima sobreposição, TDOMS. Ao contrário do caso anterior, $X$ não necessita ser um vetor de comprimento $2^{N}$, de modo que tomamos $X$ um vetor de observações para uma dada série temporal de comprimento $N$. Então, o vetor $\widetilde{w}$ de comprimento $(J+1) N$ dos coeficientes de TDOMS é dado por:

$$
\tilde{\omega}=\tilde{W} X
$$


onde $\widetilde{W}$ é uma matriz do $\mathrm{R}^{(\mathrm{J}+1) \mathrm{N}_{x} \mathrm{~N}}$ que define a TDOMS, e é construída de forma análoga àquela brevemente descrita para o caso da TDO. Deste modo, o uso das matrizes $W$ e $\widetilde{W}$ é crucial na análise da composição do sinal em cada uma das distintas escalas em cada instante do tempo.

Neste trabalho foram utilizadas as ondaletas de Daubechies, D(2), ou ondaleta de Haar, e D(8), ou L(8), todas elas ondaletas de suporte compacto. ${ }^{3}$

\subsection{Obtendo Estruturas de Correlação e Variâncias}

O objetivo principal deste trabalho é estimar uma estrutura de volatilidades e correlações para os índices financeiros descritos há pouco nas diversas escalas permitidas pela análise de ondaletas. Para tanto, apresentamos a metodologia empregada.

Considere a série temporal $X$ e seja:

$$
\left\{\tilde{h}_{j, l}: l=0, \ldots, L_{j-1}\right\}
$$

o filtro de ondaletas para a $j$-ésima escala da TDOMS, onde:

$$
L_{j}=\left(2^{j}-1\right)(L-1)
$$

é o tamanho do filtro e $L$ é o tamanho do filtro $\widetilde{h}_{l}$. Então, podemos definir o processo estocástico:

$$
\widetilde{W}_{j, t}=\sum_{l=0}^{L_{j}{ }^{-1}} \widetilde{h}_{j, l} X_{t-l}
$$

obtido pela filtragem de $X$ pelos coeficientes de ondaletas da TDOMS.

3 Para uma revisão bastante completa da teoria envolvendo ondaletas referimo-nos a [Mo99]. Para aplicações de ondaletas a problemas de séries temporais e séries temporais financeiras, referimonos aos trabalhos de [Pe83], [Pe92], [Pe93], [Pe94], [Pe95], [PeGu94], [PeMo97], [PeWa93], [PeWa00]. Para uma discussão mais completa da TODO e da TDOMS, sugere-se a leitura de [PeWa00]. 
Assim temos a:

Definição 2 - Se existir, e for finita, a variância de ondaletas tempo-dependente para a escala $\sigma_{j}$, do sinal X, é dada por:

$$
v_{X, t}^{2}\left(\sigma_{j}\right)=\operatorname{var}\left\{\tilde{W}_{j, t}\right\}
$$

Caso esta grandeza seja independente de t então, $\boldsymbol{v}_{X, t}^{2}\left(\sigma_{j}\right)=\boldsymbol{v}_{X}^{2}\left(\sigma_{j}\right)$ é chamada variância de ondaletas tempo-independente para a escala $\sigma_{j}$.

Definição 3 - Considere dois sinais X e Y de mesmo comprimento. Então a covariância de ondaletas para a escala $\sigma_{j}$ destas séries é dada por:

$$
v_{X, Y}\left(\sigma_{j}\right)=\operatorname{cov}\left\{\tilde{W}_{X, j, t}, \tilde{W}_{Y, j, t}\right\}
$$

Como é usual, esta medida pode ser normalizada para um coeficiente de correlação através de:

$$
\rho_{X, Y}\left(\sigma_{j}\right)=\frac{\operatorname{cov}\left\{\tilde{W}_{X, j, t}, \tilde{W}_{Y, j, t}\right\}}{\left(\operatorname{var}\left\{\tilde{W}_{X, j, t}\right\} \operatorname{var}\left\{\tilde{W}_{Y, j, t}\right\}\right)^{1 / 2}}=\frac{\nu_{X, Y}\left(\sigma_{j}\right)}{v_{X}\left(\sigma_{j}\right) v_{Y}\left(\sigma_{j}\right)}
$$

Apesar da possibilidade de procedermos a uma análise de variância com base nos coeficientes da TDO, nosso procedimento considera apenas a transformada discreta de ondaletas de máxima sobreposição. Isto se deve ao fato de que o estimador de variância baseado nos coeficientes da TDOMS é assintoticamente mais eficiente do que aquele baseado nos coeficientes da TDO [Pe95].

Os estimadores utilizados neste trabalho são aqueles sugeridos por [PeWa02], ao qual nos referimos para as propriedades e resultados gerais acerca destes mesmos estimadores. A estrutura de correlações e variâncias de ondaletas apresentadas nas próximas seções, bem como a análise de decomposição de ondaletas, é obtida através do software R. 


\section{Análise Exploratória de Dados}

Com a finalidade de discutirmos o comportamento das séries de retornos para os índices financeiros considerados neste trabalho, realizaremos uma análise exploratória dos dados, abordando inclusive as transformações de ondaletas TDO e TDOMS. De certa maneira, por se tratar de uma análise exploratória, não são realizados neste momento testes de hipóteses sobre as estatísticas obtidas.

Iniciamos nossa análise com a série do Ibovespa. Observemos na Figura 2 o gráfico dos retornos do Ibovespa seguido do seu gráfico quantil-quantil normal:

Retornos, Ibovespa

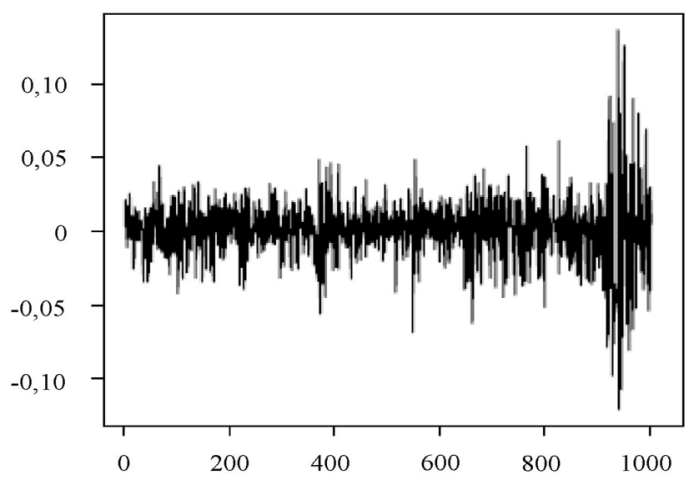

Figura 2 - Série de Retornos para o Ibovespa

Histograma, Ibovespa

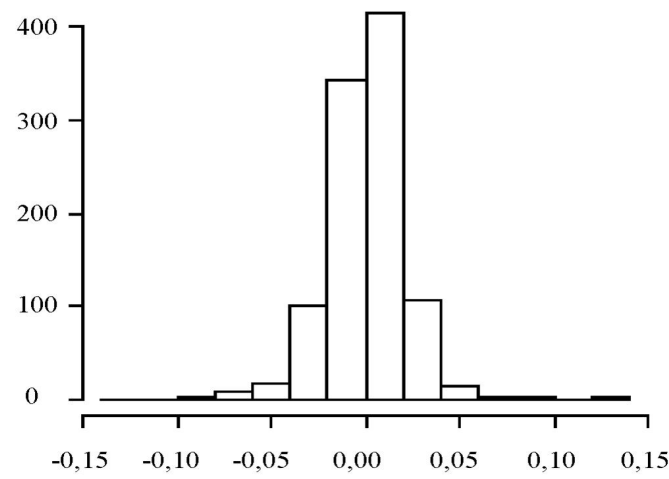

Figura 3 - Histograma, Retornos, Ibovespa 


\section{Normal, Q×Q, Ibovespa}

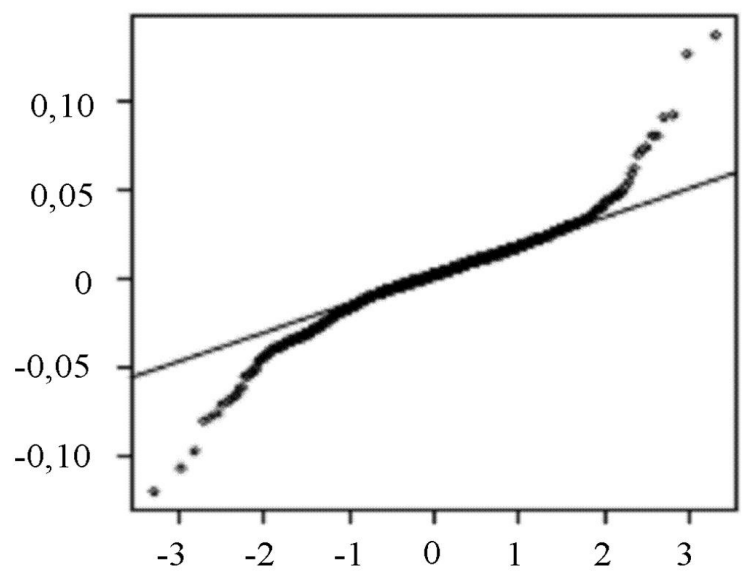

Figura 4 - Gráfico QxQ, Ibovespa

A análise da Figura 4 sugere que os retornos do Ibovespa não apresentam uma distribuição normal, além de apresentarem maior volatilidade na última terça parte do período analisado. A observação do histograma, Figura 3, sugere a presença de caudas mais pesadas do que a de uma distribuição Gaussiana padrão. Passemos à análise dos retornos para a série do Dow Jones Industrial:

Retornos, Dow Jones

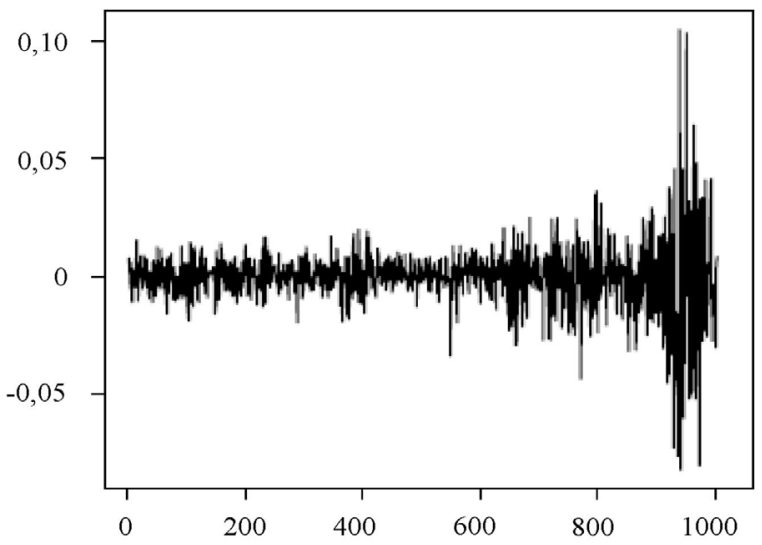

Figura 5 - Série de Retornos para o Dow Jones 
Histograma, Dow Jones

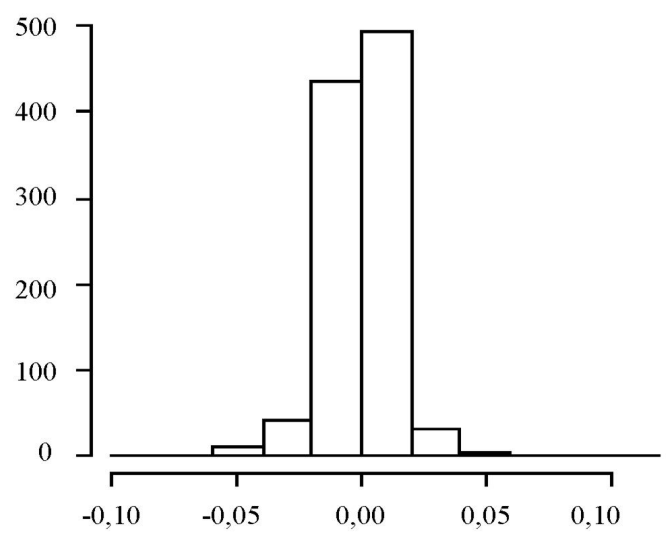

Figura 6 - Histograma, Retornos, Dow Jones

Normal, Q×Q, Dow Jones

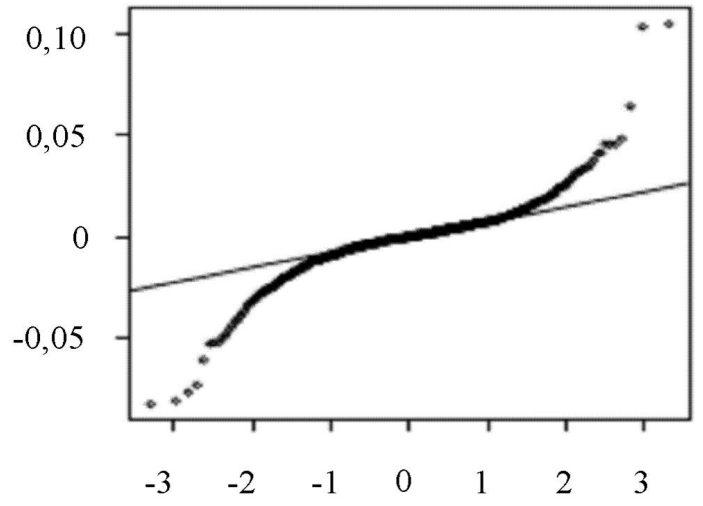

Figura 7 - Gráfico QxQ, Dow Jones

Novamente, observamos uma série de retornos com elevada oscilação no último terço do período analisado. Entretanto, esta oscilação parece mais acentuada do que no caso do Ibovespa, indicando uma volatilidade maior no período para os retornos do índice Dow Jones Industrial. Mais uma vez obtemos fortes evidências de que a distribuição destes retornos não segue uma Gaussiana padrão, uma vez que 
as caudas da distribuição sugerem um comportamento leptocúrtico. No caso dos retornos para o Euro Stoxx observamos fenômeno parecido:

Retornos, Euro Stoxx 50

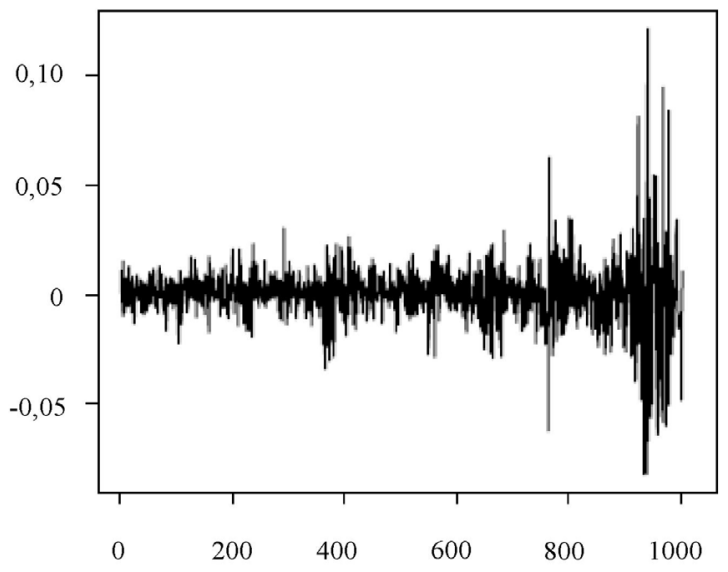

Figura 8 - Série de Retornos, Euro Stoxx 50

Histograma, Euro Stoxx 50

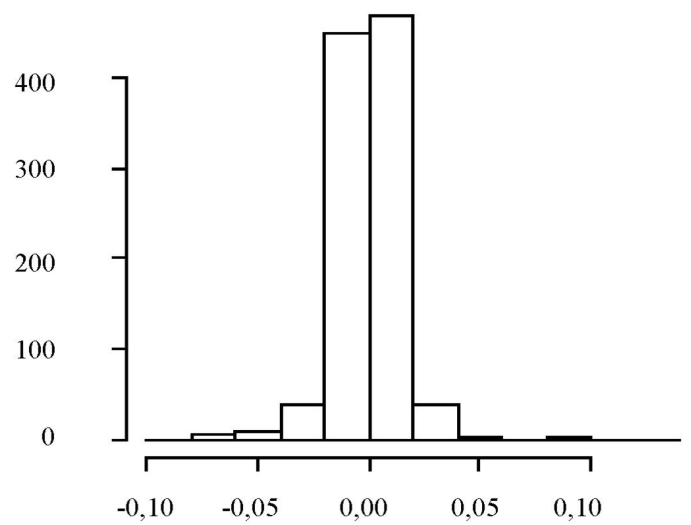

Figura 9 - Histograma, Retornos, Euro Stoxx 50 
Normal, Q×Q, Euro Stoxx 50

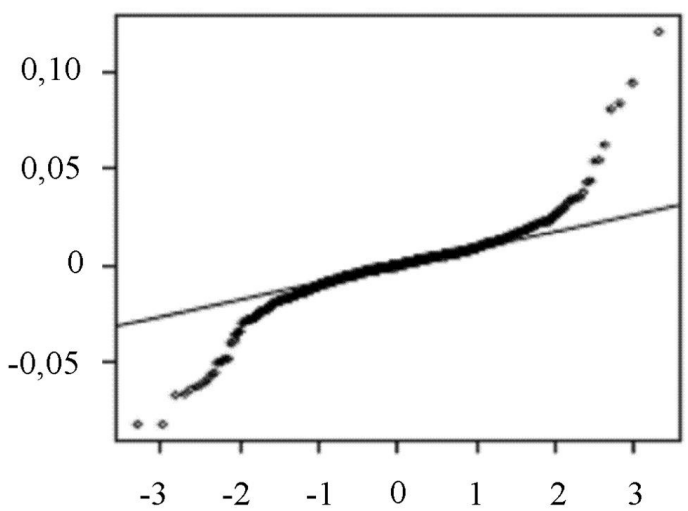

Figura 10 - Gráfico QxQ, Euro Stoxx 50

Não é surpreendente que os retornos do Euro Stoxx 50 também apresentem um comportamento bastante errático com elevação de volatilidade ao final do período de análise. Alinhando seu comportamento àqueles sugeridos pelos gráficos anteriores, esta série também oferece evidências de uma distribuição com caudas mais pesadas que aquelas realizadas por uma Normal. De modo geral, a análise exploratória sugere que as três séries apresentam comportamentos semelhantes ao longo do período analisado. Entretanto, conforme observamos anteriormente, não é possível ainda diferenciarmos os níveis de associação entre as diversas escalas ou frequências de cada sinal - esta tarefa será realizada na próxima seção:

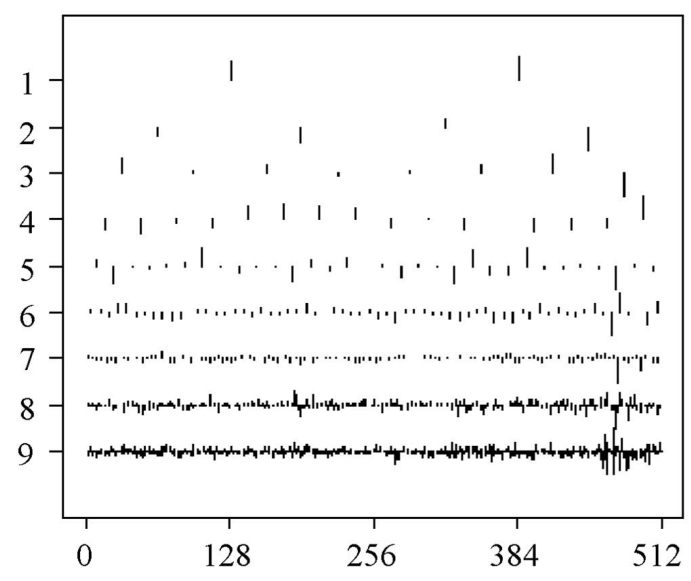

Figura 11 - TDO, Retornos, Ibovespa 


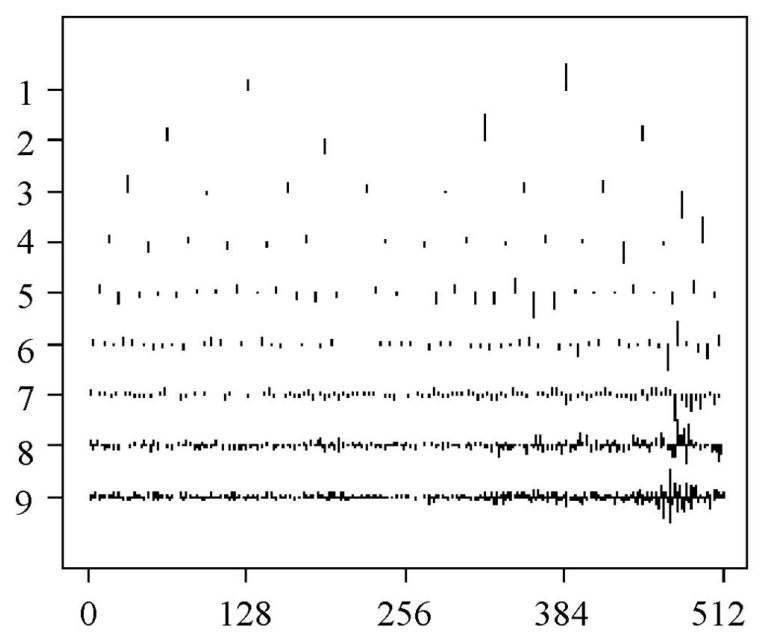

Figura 12 - TDO, Retornos, Dow Jones

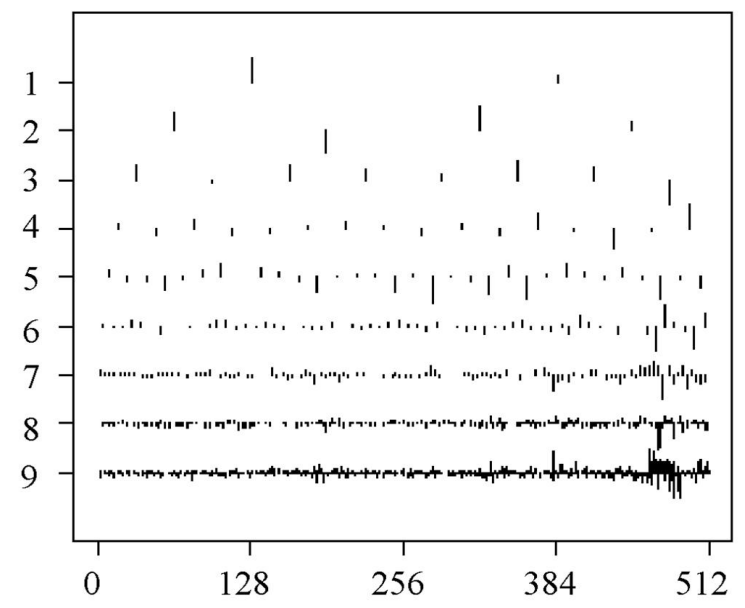

Figura 13 - TDO, Retornos, Euro Stoxx 50

Passemos agora à análise dos coeficientes de ondaletas obtidos pela TDO. Para as três séries os coeficientes referentes ao movimento de investimentos de longo prazo apresentam magnitude superior a dos coeficientes relativos ao investimento de curto prazo. No período correspondente aos coeficientes entre 450 e 500 , as três primeiras escalas apresentam significantes magnitudes, o que indica uma perturbação considerável dos investimentos de curto prazo no período: 


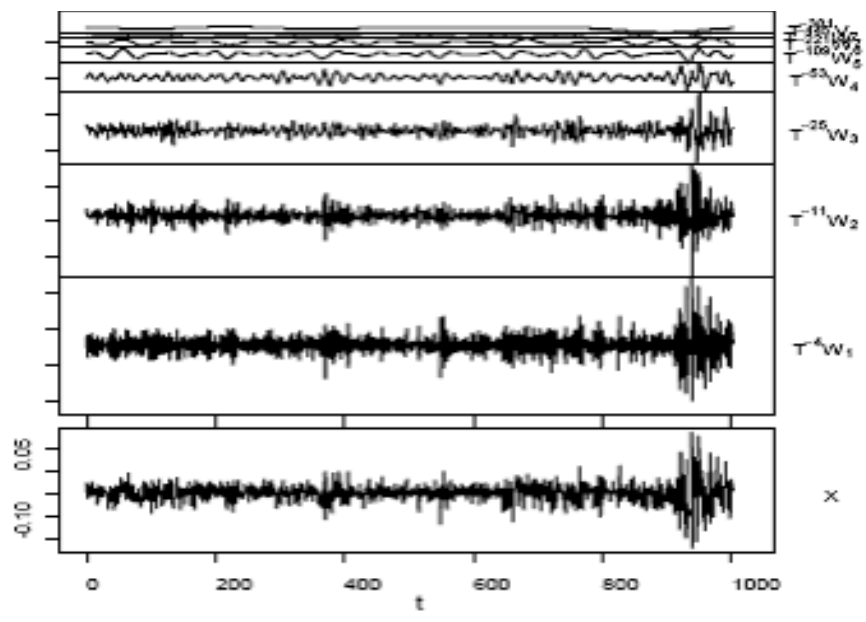

Figura 13 - Análise de Multiresolução, Retornos, Ibovespa

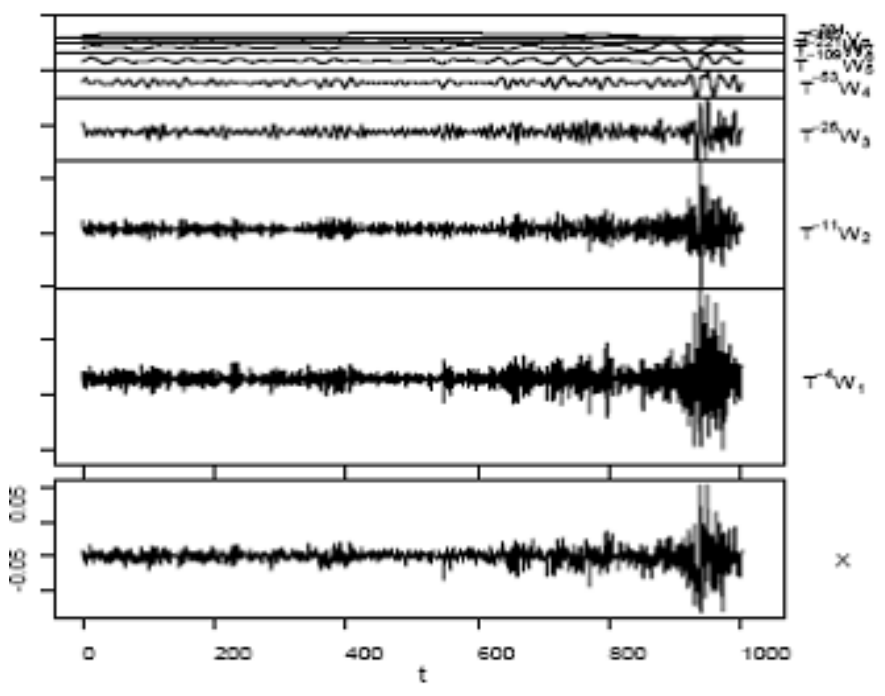

Figura 14 - Análise de Multiresolução, Retornos, Dow Jones 


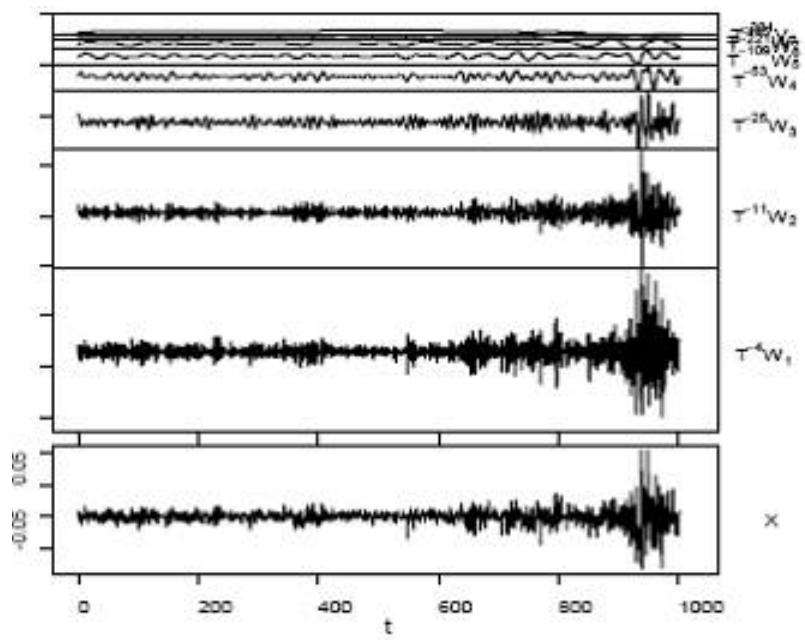

Figura 15 - Análise de Multiresolução, Retornos, Euro Stoxx 50

Da mesma forma, ao abordarmos a análise de multiresolução com base na TDOMS para as três séries, notamos que as frequências que mais contribuem com a energia de cada sinal são as três frequências mais altas. Desta forma, sugerimos a interpretação de que para os três índices, os investimentos de curto prazo são preponderantes em sua composição e movimento. Novamente, verifica-se uma flutuação largamente mais acentuada no período que coincide com a crise recente dos mercados financeiros nestas mesmas frequências, o que oferece consistência à conclusão de que os investimentos cuja volatilidade é mais acentuada no contexto da crise financeira são aqueles de curto prazo.

Já para o caso de escalas mais altas - investimento de longo prazo - além de estes contribuírem de forma limitada para a composição do sinal, não há nenhuma oscilação que mereça destaque em todo o período de análise.

\section{Resultados e Discussão}

Nesta seção apresentaremos as matrizes de variância de cada série de retornos, seguidas das matrizes de correlação, e discutiremos seus resultados, oferecendo sua interpretação econômica. Todos os valores reportados são significantes a 5\%, como usualmente, e, para a análise de correlação, as três últimas escalas não apresentaram resultados estatisticamente significantes. Em cada tabela, $d_{j}$ representa a $j$-ésima escala de ondaleta, onde $j=1, \ldots, 7$. Quanto mais baixo for $j$, mais alta a frequência da análise. 
Tabela 1 - Retornos Ibovespa

\begin{tabular}{lccc}
\hline \multicolumn{3}{c}{ Análise de Volatilidade } \\
\hline$d_{1}$ & $2,332 \times 10^{-4}$ & $d_{6}$ & $3,539 \times 10^{-6}$ \\
$d_{2}$ & $1,375 \times 10^{-4}$ & $d_{7}$ & $8,534 \times 10^{-7}$ \\
$d_{3}$ & $d_{8}$ & $\sim 10^{-10}$ \\
$d_{4}$ & $6,653 \times 10^{-5}$ & $d_{9}$ & $\sim 10^{-10}$ \\
$d_{5}$ & $2,071 \times 10^{-5}$ & $d_{10}$ & $\sim 10^{-10}$ \\
\hline
\end{tabular}

Tabela 2 - Retornos Euro Stoxx

\begin{tabular}{lccc}
\hline \multicolumn{3}{c}{ Análise de Volatilidade } \\
\hline$d_{1}$ & $1,220 \times 10^{-4}$ & $d_{6}$ & $1,434 \times 10^{-6}$ \\
$d_{2}$ & $d_{7}$ & $2,738 \times 10^{-7}$ \\
$d_{3}$ & $2,870 \times 10^{-5}$ & $d_{8}$ & $\sim 10^{-10}$ \\
$d_{4}$ & $2,435 \times 10^{-5}$ & $d_{9}$ & $\sim 10^{-10}$ \\
$d_{5}$ & $8,925 \times 10^{-6}$ & $d_{10}$ & $\sim 10^{-10}$ \\
\hline
\end{tabular}

Tabela 3 - Retornos Dow Jones Industrial

\begin{tabular}{lccc}
\hline \multicolumn{3}{c}{ Análise de Volatilidade } \\
\hline$d_{1}$ & $d_{6}$ & $7,160 \times 10^{-7}$ \\
$d_{2}$ & $1,143 \times 10^{-4}$ & $d_{7}$ & $3,337 \times 10^{-7}$ \\
$d_{3}$ & $4,202 \times 10^{-5}$ & $d_{8}$ & $\sim 10^{-10}$ \\
$d_{4}$ & $1,834 \times 10^{-5}$ & $d_{9}$ & $\sim 10^{-10}$ \\
$d_{5}$ & $6,242 \times 10^{-6}$ & $d_{10}$ & $\sim 10^{-10}$ \\
\hline
\end{tabular}

Para todas as séries de retornos analisadas, a volatilidade decresce conforme a escala aumenta, ou seja, os componentes de mais alta frequência dos índices analisados apresentam maior volatilidade. Este resultado satisfaz às expectativas econômicas que atribuem ao investimento de mais alta frequência um grau de risco mais elevado. Mais interessante, entretanto, é o fato de que em cada diferente escala, os retornos do Ibovespa apresentaram a mais alta volatilidade, seguido dos retornos do Euro e, por fim, do menos volátil retorno Dow Jones Industrial. Este fato corrobora, por assim dizer, a noção de uma volatilidade mais baixa no mercado americano - tão chamado livre de risco - seguida do mercado europeu e de um grau de incerteza mais elevado no mercado brasileiro. De modo geral, os resultados indicam maiores níveis de estabilidade nos mercados americano e europeu, no que diz respeito a investimentos de curto prazo, na medida em que investimentos de longo prazo possuem graus de volatilidade semelhantes. 
Passemos agora à análise de correlação de ondaletas para estas mesmas séries de retornos, nas mesmas dez escalas temporais distintas.

\section{Tabela 4 - Correlação de Ondaletas}

\begin{tabular}{lccc}
\hline$d_{1}$ & Ibovespa & Euro Stoxx & Dow Jones \\
Ibovespa & 1,000 & 0,444 & 0,603 \\
Euro Stoxx & 0,444 & 1 & 0,302 \\
Dow Jones & 0,603 & 0,302 & 1,000 \\
\hline$d_{2}$ & Ibovespa & Euro Stoxx & Dow Jones \\
Ibovespa & 1,000 & 0,623 & 0,688 \\
Euro Stoxx & 0,623 & 1 & 0,582 \\
Dow Jones & 0,688 & 0,582 & 1,000 \\
\hline$d_{3}$ & Ibovespa & Euro Stoxx & Dow Jones \\
Ibovespa & 1,000 & 0,689 & 0,744 \\
Euro Stoxx & 0,689 & 1 & 0,781 \\
Dow Jones & 0,744 & 0,781 & 1,000 \\
\hline$d_{4}$ & Ibovespa & Euro Stoxx & Dow Jones \\
Ibovespa & 1,000 & 0,737 & 0,670 \\
Euro Stoxx & 0,737 & 1 & 0,810 \\
Dow Jones & 0,670 & 0,810 & 1,000 \\
\hline$d_{5}$ & Ibovespa & Euro Stoxx & Dow Jones \\
Ibovespa & 1,000 & 0,731 & 0,700 \\
Euro Stoxx & 0,731 & 1 & 0,764 \\
Dow Jones & 0,700 & 0,764 & 1,000 \\
\hline$d_{6}$ & Ibovespa & Euro Stoxx & 0,557 \\
Ibovespa & 1,000 & 0,591 & 0,809 \\
Euro Stoxx & 0,591 & 1 & 0,000 \\
\hline Dow Jones & 0,557 & 0,809 & 1,000 \\
\hline$d_{7}$ & Ibovespa & \\
Ibovespa & 1,000 & Duro Stoxx & \\
Euro Stoxx & 0,704 & 0,704 & \\
Dow Jones & 0,864 & 1 & \\
\hline & & & \\
\hline
\end{tabular}

A análise da Tabela 4 acima indica que, para todas as escalas temporais, a correlação entre os retornos do Ibovespa, Euro Stoxx e Dow Jones Industrial, dois a dois, é positiva. Tomando-se o Ibovespa e o Dow Jones Industrial, podemos ver que para as três primeiras escalas - com frequências baixas - estes mercados parecem bastante 
correlacionados e de forma crescente nestas mesmas escalas. Isto pode sugerir que movimentos nos retornos, devido ao comportamento de investimentos de mais baixa frequência - que já identificamos com o micro e pequeno investimento, p.e. -, estão altamente correlacionados em ambos os mercados. Em escalas mais altas, ambos os índices voltam a apresentar correlações crescentes, indicando a possibilidade de uma aderência maior entre os mercados no que diz respeito ao investimento de longo prazo.

Já os mercados europeu e americano apresentam baixa correlação na primeira escala. Entretanto, essa cresce rapidamente e atinge níveis muito próximos de 1 na sétima escala. Este fato dá suporte à ideia de que, apesar dos investimentos de altíssima frequência apresentarem algum grau de descolamento em ambos os mercados, à medida que esta mesma frequência diminui, estes mercados apresentam comportamentos muito semelhantes.

\section{Comentários Finais}

Este artigo implementa uma análise de ondaletas para indicadores financeiros representativos da performance dos mercados americano, brasileiro e europeu - em dois níveis, no período dos últimos cinco anos, (2004-2009). A princípio, analisando padrões particulares de cada série e, posteriormente, procedendo a uma análise de volatilidade e correlação com base em decomposição de ondaletas.

Em um primeiro nível, pudemos constatar que os investimentos de curto prazo - ou de frequência mais alta - foram os que mais contribuíram com a formação de cada sinal, bem como apresentaram maior flutuação no período recente. Este grupo de investimento representa majoritariamente o investimento especulativo. Já os investimentos de longo prazo, ou de frequência mais baixa, além de sofrerem menores oscilações, ainda exerceram menor contribuição - energia - à formação dos índices. Este grupo de investimentos é representado principalmente pela poupança dos indivíduos, micro e pequenos investidores, bem como de depósitos a prazo.

No que diz respeito à análise de volatilidade, o mercado brasileiro foi o que apresentou os mais elevados níveis de instabilidade, desde as mais baixas até as mais altas frequências. Entretanto, esta volatilidade é decrescente nas escalas: investimentos de longo prazo são menos voláteis do que aqueles de curto prazo. Em seguida, em um nível intermediário de volatilidade aparece o mercado europeu. Neste caso, observamos o mesmo comportamento decrescente nas escalas. No entanto, no caso do mercado europeu, a queda na volatilidade é mais acentuada do que no caso brasileiro; em relação às três primeiras escalas, a queda de volatilidade no mercado 
europeu é cerca de 10\% superior àquela verificada no caso brasileiro. Já o mercado americano possui os mais baixos níveis de volatilidade, que são decrescentes na medida em que a frequência dos investimentos diminui.

Em comparação com o mercado europeu, os níveis de volatilidade americanos caem cerca de 12\% mais rápido ao mudarmos da primeira para a segunda escala. Já com relação ao mercado brasileiro, este número salta para 22\%. Em geral, os índices de volatilidade do mercado brasileiro são mais elevados e caem mais devagar do que aqueles para o mercado europeu. Situação semelhante verifica-se ao compararmos o mercado europeu e americano, sendo que, neste caso, o que apresenta níveis mais baixos de volatilidade com quedas mais acentuadas é o americano.

A análise de correlações apontou grau positivo para todos os mercados, em todas as escalas. Entretanto, no que tange a investimentos de longo prazo, esta correlação é mais alta do que aquela verificada para investimentos de curto prazo.

Desta maneira, encontram-se evidências de que os três mercados apresentam algum grau de interdependência; entretanto, observa-se que esta inter-relação é mais acentuada no caso dos mercados de investimento de longo prazo. Este fato sugere que o grau de instabilidade encontrado pelo micro e pequeno investidor é muito semelhante dentre os diversos mercados.

Por outro lado, a menor correlação verificada para os graus de volatilidade dos mercados de curto prazo sugere que, apesar da interdependência engendrada por fatores como a mobilidade de capital, alguns fatores pertinentes a cada economia - como a estabilidade e desempenho de seus agregados macroeconômicos ou seu panorama institucional e regulatório - podem ser elementos determinantes da estabilidade de seu mercado financeiro.

\section{Referências}

[BrDe05] BROOKS, R.; DEL NEGRO, M. Country versus region effects in international stock returns, Journal of Portfolio Management, 67-72, Summer. 2005.

[BrDe06] BROOKS, R.; DEL NEGRO, M. Firm-level evidence on international stock market comovement, Review of Finance, 10, 69-98. 2006.

[Da92] DAUBECHIES, I. Ten lectures on wavelets, Volume 61 of CBMS-NSF Regional Conference Series in Applied Mathematics. Society for Industrial and Applied Mathematics, Philadelphia. 1992. 
[FoRi02] FORBES, K.; Rigobon, R. No contagion, only interdependence: measuring stock market comovements, Journal of Finance, 57, 2223-2261. 2002.

[Gr68] GRUBEL, H. Internationally diversified portfolios: welfare gains and capital Flows, American Economic Review, 58, 1299-1314. 1968.

[Ha 10] HAAR, A. Zur theorie der orthogonalen funktionensysteme. Mathematische Annalen, 69, 331-371. In German. 1910.

[KaSt96] KAROLYI, G. A; STULZ, R. M. Why do markets move together? An investigation of U.S.-Japan stock return comovements, Journal of Finance, 51, 951-986. 1996.

[Ki94] KING, M.; SENTANA, E.; SUSHIL, W. Volatility and links between national stock markets, Econometrica, 62, 668-675. 1994.

[LeSa70] LEVY, H.; SARNAT, M. International diversification of investment portfolios, American Economic Review, 60, 668-675. 1970.

[Li94] LIN, W.-L.; ENGLE, R.; ITO, T. Do bulls and bears move across borders? International transmission of stock returns and volatility, Review of Financial Studies, 7, 507-538. 1994.

[LoSo95] LONGIN, F.; SOLNIK, B. Is the correlation of international equity returns constant: 1960-1990, Journal of International Money and Finance, 14, 3-26. 1995.

[LoSo01] LONGIN, F.; SOLNIK, B. Extreme correlation of international equity markets, Journal of Finance, 56, 649-676. 2001.

[Mo99] MORETTIN, P. A. Ondas e ondaletas, da análise de Fourier à análise de ondaletas. São Paulo. Edusp. 1999.

[Pe83] PERCIVAL, D. B. The statistics of long memory processes. Ph.D. thesis, Department of Statistics, University of Washington. 1983.

[Pe92] PERCIVAL, D. B. Simulating Gaussian random processes with specified spectra. Computing Science and Statistics, 24, 534-538. 1992.

[Pe93] PERCIVAL, D. B. Three curious properties of the sample variance and autocovariance for stationary processes with unknown mean. The American Statistician, 47, 274-276. 1993.

[Pe94] PERCIVAL, D. B. Spectral analysis of univariate and bivariate time series. In J. L. STANFORD AND S. B. VARDEMAN, editors, Statistical Methods for Physical Science, Volume 28 of Methods of Experimental Physics, 313-348. Academic press, Inc., Boston. 1994.

[Pe95] PERCIVAL, D. B. On estimation of the wavelet variance. Biometrika, 82, 619-631. 1995.

[PeGu94] PERCIVAL, D. B.; GUTTORP, P. Long-memory processes, the Allan variance and wavelets. In E. FOUFOULA-GEORGIOU and P. KUMAR, editors, Wavelets in Geophysics, Volume 4 of Wavelet Analysis and Its Applications, 325-344. Academic Press, Inc, San Diego. 1994. 
[PeMo97] PERCIVAL, D. B. and MOFJELD, H. O. Analysis of subtidal coastal sea level fluctuations using wavelets. Journal of the American Statistical Association, 92, 868-880. 1997.

[PeWa93] PERCIVAL, D. B.; WALDEN, A. T. Spectral analysis for physical applications: multitaper and conventional univariate techniques. Cambridge University Press, Cambridge, England. 1993.

[PeWa00] PERCIVAL, D. B.; WALDEN, A. T. Wavelet methods for time series analysis. Cambridge University Press, Cambridge. 2000.

[RuNu09] RUA, A.; NUNES, L. C. International comovement of stock market returns: a wavelet analysis, Journal of Empirical Finance. 2009. 